\title{
Does media multitasking always hurt? A positive correlation between multitasking and multisensory integration
}

\author{
Kelvin F. H. Lui • Alan C.-N. Wong
}

Published online: 12 April 2012

(C) Psychonomic Society, Inc. 2012

\begin{abstract}
Heavy media multitaskers have been found to perform poorly in certain cognitive tasks involving task switching, selective attention, and working memory. An account for this is that with a breadth-biased style of cognitive control, multitaskers tend to pay attention to various information available in the environment, without sufficient focus on the information most relevant to the task at hand. This cognitive style, however, may not cause a general deficit in all kinds of tasks. We tested the hypothesis that heavy media multitaskers would perform better in a multisensory integration task than would others, due to their extensive experience in integrating information from different modalities. Sixty-three participants filled out a questionnaire about their media usage and completed a visual search task with and without synchronous tones (pip-and-pop paradigm). It was found that a higher degree of media multitasking was correlated with better multisensory integration. The fact that heavy media multitaskers are not deficient in all kinds of cognitive tasks suggests that media multitasking does not always hurt.
\end{abstract}

Keywords Attention $\cdot$ Media multitasking

\section{Introduction}

Humans often engage in multitasking - that is, performing more than one task simultaneously or in rapid alternation. We can effortlessly talk to each other while walking and watch television while eating. Certain multitasking situations are

K. F. H. Lui $(\varangle) \cdot$ A. C.-N. Wong

Department of Psychology,

The Chinese University of Hong Kong,

362A Sino Building,

Shatin, NT, Hong Kong

e-mail: f2a8kelvin@yahoo.com.hk more challenging because the tasks involved are more difficult or they concurrently engage overlapping processes and, thus, interfere with each other more. Talking on cell phones while driving is a good example (e.g., Nunes \& Recarte, 2002; Strayer \& Johnston, 2001). Sometimes, such difficulties can have serious consequences.

One way that modern technology has transformed human living styles is through the invention and popularization of various media of communication and interaction, including radio, television, telephone and text messaging, video games, computers, and the Internet. Media multitasking has thus grown into a popular phenomenon and even a way of life, especially among young people (Roberts, Foehr, \& Rideout, 2005). The high accessibility of computers, in particular, has cultivated obsessive multitasking, due to the ease of switching between instant messaging, music, Web surfing, e-mail, online videos, computer-based games, computer-based applications, and so forth (Foehr, 2006).

The immediate effects of media multitasking on one's memory, learning, and other cognitive functions have been a focus of recent research. It was found that watching TV while doing homework decreased participants' performance on the recognition of TV content (Zhang, Jeong, \& Fishbein, 2010). Besides, Furnham and Bradley (1997) found detrimental effects of background music on participants' performance in various cognitive tasks. Armstrong and Chung (2000) showed a negative effect of background television during science article reading on subsequent recall and recognition. Similarly, reading and watching video simultaneously impaired participants' performance on the reading task (Lin, Robertson, \& Lee, 2009). Moreover, Hembrooke and Gay (2003) found that opening laptops and engaging in media multitasking behaviors during a lecture impaired students' memory for lecture contents.

Research also has examined the chronic effects of media multitasking. Kirschner and Karpinski (2010) found that heavy 
Facebook users reported having lower grade point averages (GPAs) and suggested the possible deleterious effect of simultaneously using Facebook with other activities. Besides, the amount of time spent on instant messaging was found to be negatively correlated both with cognitive performance in lab tasks and with GPA (Fox, Rosen, \& Crawford, 2009). While these two studies assumed that Facebook usage and instant messaging often co-occur with other activities and, thus, represent multimedia usage, they did not, however, exclude the simpler possibility that Facebook usage and instant messaging simply occupy the time that could be used for studying.

A recent large-scale study (Ophir, Nass, \& Wagner, 2009) compared the way in which heavy media multitaskers process information differently from those who do not. Participants completed a self-report questionnaire in their study to indicate their level of media multitasking. It was found that heavy media multitaskers performed worse than light media multitaskers in several cognitive tasks that involved working memory, selective attention to task-relevant information in visual search, and surprisingly, task switching. It was suggested that heavy media multitaskers have developed the habit of simultaneously consuming information from multiple media and, thus, "breadth-biased cognitive control." As a result, heavy media multitaskers could not precisely differentiate between important information and distractors. They readily spread their attention to different sources of information regardless of whether they were relevant to the primary task at hand. This is why they were inferior to light media multitaskers in tasks that required one to disregard irrelevant information in the environment and in memory.

An intriguing possibility, however, is that their breadthbiased cognitive control will lead to better performance if a task contains some unexpected information that is important to the task at hand. When a media multitasker reads, for example, he or she may more readily detect an SMS ringtone from a mobile phone, although the ringtone does not carry information useful for the primary task of reading. In fact, situations like that may actually be more representative of what happens in real life (Lin, 2009).

In this study, our aim was to measure the potential difference between high and low media multitaskers in this tendency to capture information from seemingly irrelevant sources. Specifically, we accessed how much one can integrate visual and auditory information automatically, using a visual search task in the pip-and-pop paradigm (Van der Burg, Olivers, Bronkhorst, \& Theeuwes, 2008). In this paradigm, participants made speeded responses to indicate which of a horizontal or vertical line segment was present among a number of oblique line segments of various orientations. Both target and distractor lines in the display changed color between red and green continuously. Participants performed the same search task on both tone-absent (baseline) and tone-present trials, but only in tone-present trials was each target color change accompanied by a short auditory tone (the pip). Although the tone did not contain any information about the target color, location, or orientation, its synchrony did make the target pop out from the cluttered display, leading to better performance in the tone-present than in the toneabsent condition. Interestingly, as was shown in Van der Burg et al., this pip-and-pop effect is largely bottom-up and automatic, occurring even when the pip is rather uninformative (i.e., synchronous with the target only $20 \%$ of the time). These features render the pip-and-pop effect a good tool for investigating the association between media multitasking experience and one's tendency to automatically attend to and integrate seemingly task-irrelevant, unexpected sources of information.

\section{Method}

\section{Participants}

Sixty-three participants ( 34 females), from 19 to 28 years of age, participated in the experiment, with monetary compensation. All had normal hearing and normal or corrected vision.

\section{Apparatus}

A media multitasking questionnaire identical to the one in Ophir et al. (2009) was introduced to all participants. Part one asks about the number of hours one spends each week in using 11 primary media, including print media, television, computer-based video, music, nonmusic audio, video or computer games, telephone and mobile phone voice calls, instant messaging, text messaging, e-mail, Web surfing, and other computer-based applications. Part two requires the participant to fill in a media multitasking matrix with the number $0,1,2$, or 3 to indicate how often one uses each of the other media at the same time with a primary medium. A formula was used to calculate, for each participant, a media multitasking index that indicated the mean number of media the participant was engaged in concurrently. For details of the media multitasking index formula, please refer to Ophir et al.

A personal computer with E-Prime version 1.2 and a headphone were used to run the visual search task. The program was modified from the one used in Van der Burg et al.'s (2008) Experiment 1, with a few changes made to the set size and number of trials.

\section{Procedure}

All participants first filled out the media use questionnaire and then completed the search task. They were seated approximately $80 \mathrm{~cm}$ away from the screen and wore headphones during the search task. Each trial began with a fixation dot presented at the center of the screen that lasted for $1,000 \mathrm{~ms}$, 
followed by a target line and 47 distractor lines. Participants were asked to fixate on the dot during their search for the target (a horizontal or a vertical line segment). Figure 1 shows an example of the visual display used in this study. The 48 line segments were in various orientations and changed color between red and green at random intervals varied among 50,100, and $150 \mathrm{~ms}$. The target also changed its color, at an average frequency of $1.11 \mathrm{~Hz}$, but the change was asynchronous to other line segments, which meant that the other line segments never changed their colors at the same time as the target. Participants were asked to press the "m" or " $\mathrm{z}$ " button as quickly and accurately as possible to indicate whether a horizontal or a vertical target, respectively, had been presented.

There were four blocks of 30 trials each, with the first and third being tone-absent blocks in which no sound was presented. The second and final blocks were tone-present blocks in which each target color change was accompanied by a short auditory pip without time lag. The pip contained no information about the visual target's location or orientation but indicated the change instant of the target line segment. Participants were not informed of the meaning of the tone and were not told to attend to the tone. Feedback about their overall mean accuracy and reaction time (RT) was provided after each block. Two short practice blocks of 10 trials each, including one tone-absent block and one tone-present block, were introduced at the beginning of the task.

\section{Results}

The distribution of the media multitasking index (MMI) is shown in Fig. 2. The mean MMI was 3.82, while its standard deviation was 1.54 .

For the search task, trials with an RT three standard deviations over the mean for each participant were excluded for

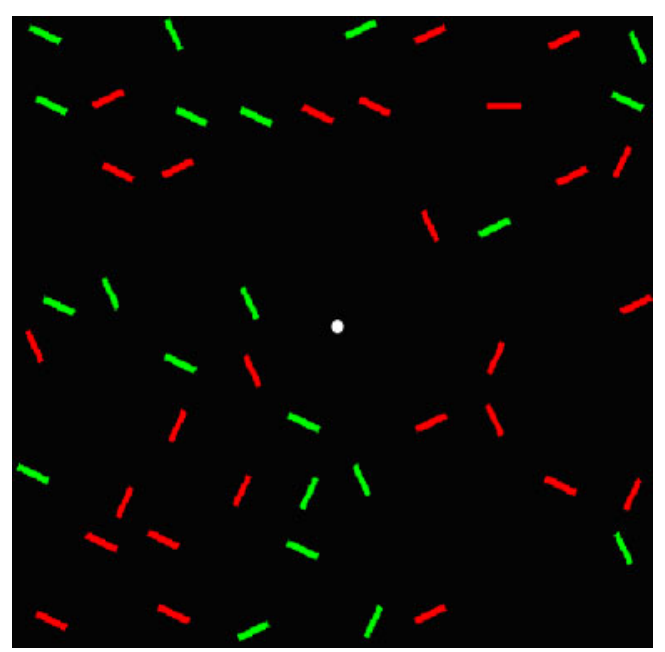

Fig. 1 An example of the visual search display used in the present study analysis. Also, the data for 3 participants were discarded because their overall accuracies were more than three standard deviations below the mean for the other participants. In calculating the mean RT, only trials with correct responses were included. The mean RTs and accuracy in the tone-absent and tone-present conditions are shown in Fig. 3. Consistent with Van der Burg et al.'s (2008) findings, performance was better in the tone-present than in the tone-absent condition, with shorter RTs, $t(59)=4.695, p<.001$, and higher accuracy, $t(59)=-1.929, p=.059$.

We also examined the relationship between the degree of media multitasking, as indicated by the MMI, and multisensory integration performance, as indicated by the facilitation of the tone in the visual task in terms of RT [(RT_no-tone $\mathrm{RT}$ _tone $) / \mathrm{RT}$ no-tone] and accuracy [(AC_tone - AC_notone)/AC_no-tone]. Pearson product correlation analyses were conducted between the two measures, with three outliers excluded in RT analysis and one in accuracy analysis due to a deviation of over three standard deviations away from the average for all participants. A positive correlation was found between the MMI and multisensory integration index in terms of accuracy, $r(57)=.292, p<.05$ (Fig. 4a). The correlation was not significant for RT, $r(55)=-.091$, $p=.50$. Overall, participants with a stronger media multitasking habit did show a larger multisensory integration effect as shown in the pip-and-pop paradigm. It should be noted that the overall media consumption hours was not correlated with the multisensory integration index in terms of accuracy, $r(57)=-.042, p=.751$, and RT, $r(55)=.017, p=.898$.

Apart from the correlation analyses, we further tested whether there was a difference in the multisensory integration effect between extreme groups of media multitaskers. Participants whose media multitasking index was one standard deviation or more above the mean were regarded as heavy media multitaskers, while those with an index one standard deviation or more below the mean were regarded as light media multitaskers. Results were consistent with the correlation analyses (Fig. 4b), with heavy media multitaskers $(N=$ $10, M=3.86 \%$ ) having a larger multisensory integration effect in terms of accuracy than did light media multitaskers $(N=9$, $M=-0.21 \%), t(17)=2.132, p<.05$. No group difference was found in terms of the improvement of RT (heavy vs. light media multitasks, $21.25 \%$ vs. $23.22 \%, t<1$ ).

Since the multisensory integration index was calculated by dividing the difference in performance between the tonepresent and tone-absent conditions by the baseline performance in the tone-absent condition, one might question whether the difference in baseline performance between heavy media multitaskers and light media multitaskers was the primary cause of the present finding. Although it was found that the heavy media multitaskers tended to be less accurate in the tone-absent condition than the light media multitaskers, $r(57)=-.369, p<.01$ (Fig. 5a), such a base 
Fig. 2 Distribution of the media multitasking index (MMI) among participants

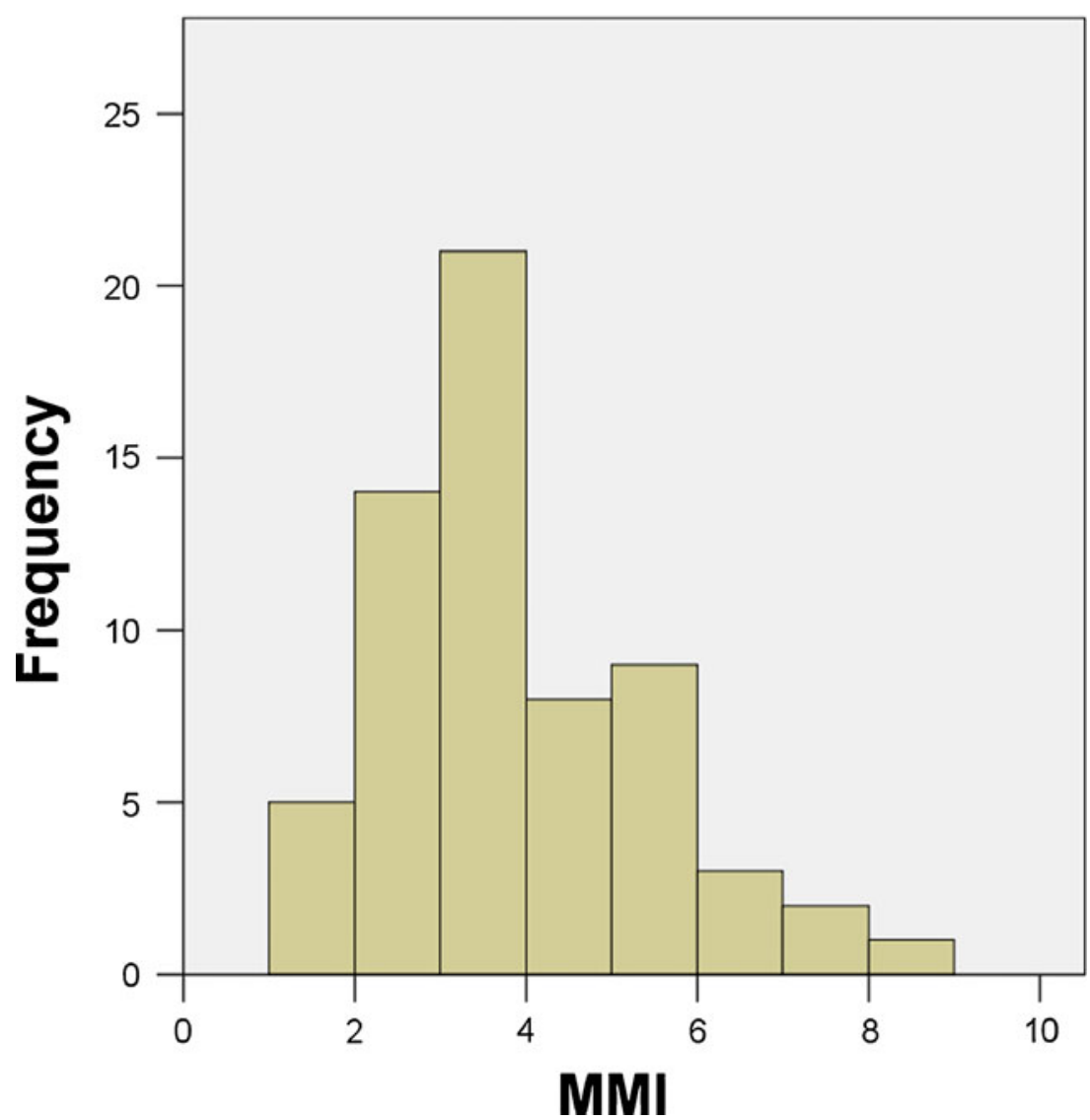

rate difference was unlikely to have been the cause of their larger percentage of improvement across the conditions. When the multisensory integration index was calculated without dividing by the baseline performance, similar results were obtained, both in terms of the positive correlation between the MMI and the multisensory integration index in accuracy, $r(57)=.284, p<.05$, and in terms of the larger multisensory integration effect for the heavy media multitaskers in the between-group analyses, $t(17)=2.123, p<.05$.
Participants' average accuracy in the tone-absent condition was quite high ( $>95 \%)$; as a result, ceiling effect could be a concern in the present study. Figure $5 \mathrm{a}-\mathrm{c}$ shows the original correlation between participants' MMI and their accuracy in the tone-absent condition, in the tone-present condition, and on the multisensory integration index, respectively. In the tone-absent condition (Fig. 5a), heavy media multitaskers performed significantly worse than light media multitaskers in the visual search task, while in the tone-present condition (Fig. 5b), such a
Fig. 3 Participants' mean reaction times and accuracy in the pip-and-pop task. Error bars indicate standard errors of the means

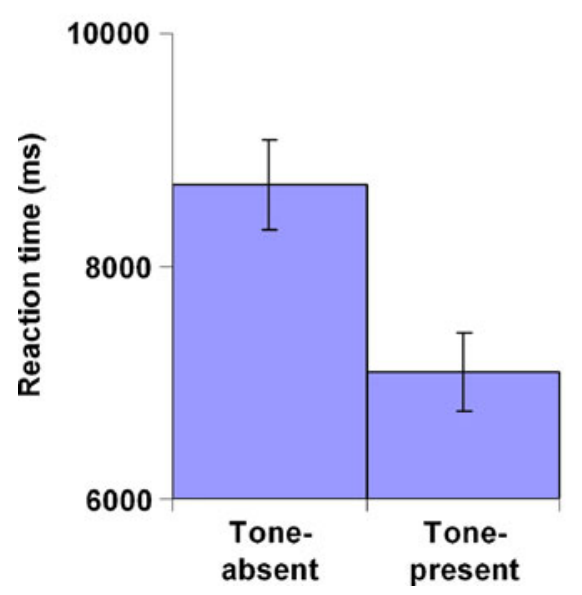

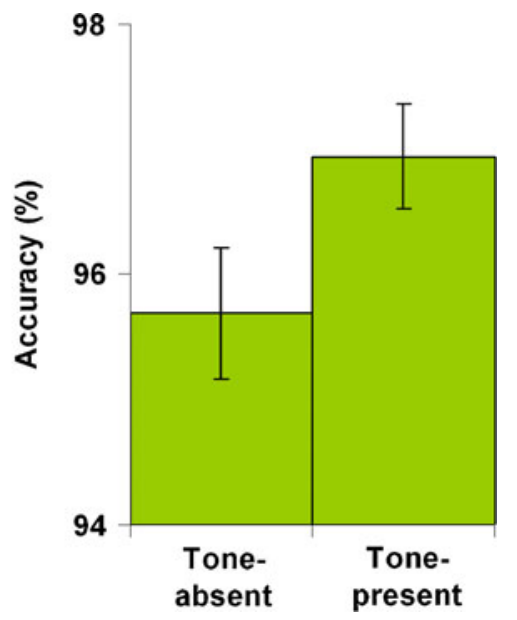


Fig. 4 a Scatterplot between the multisensory integration index for accuracy and the media multitasking index. $\mathbf{b}$ Multisensory integration indices for light and heavy media multitaskers. Error bars indicate standard errors of the means

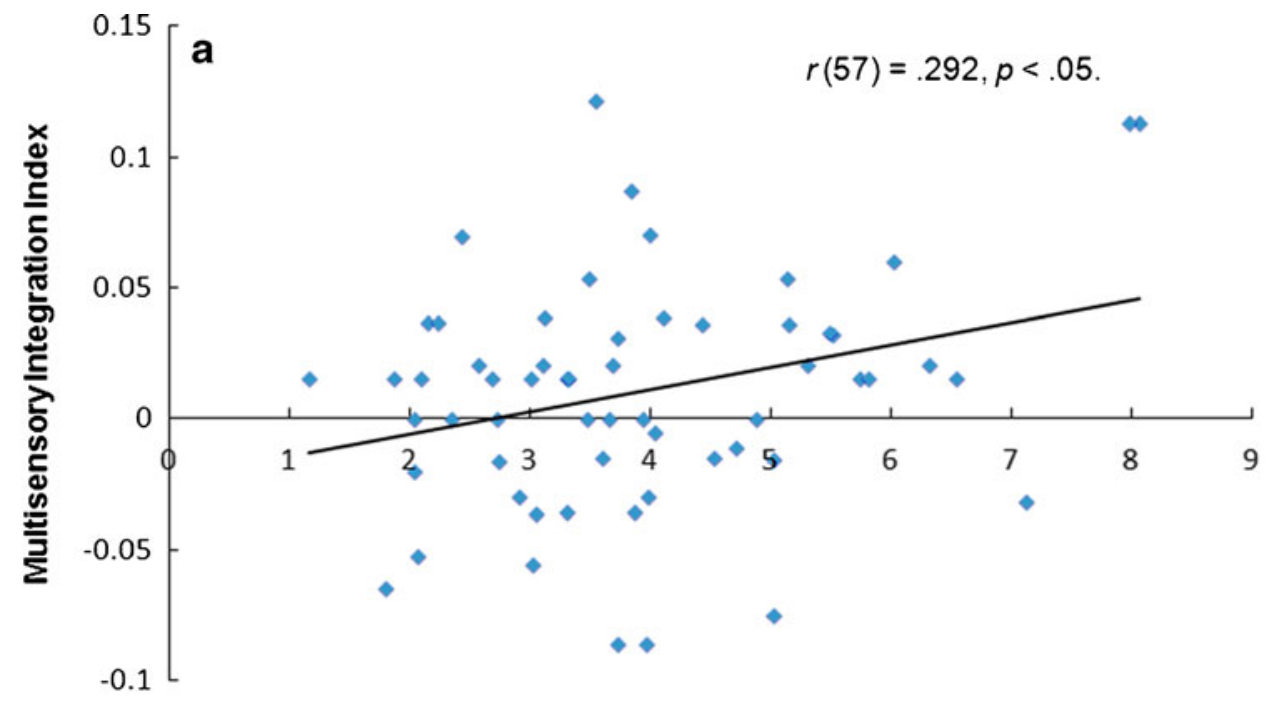

Media Multitasking Index

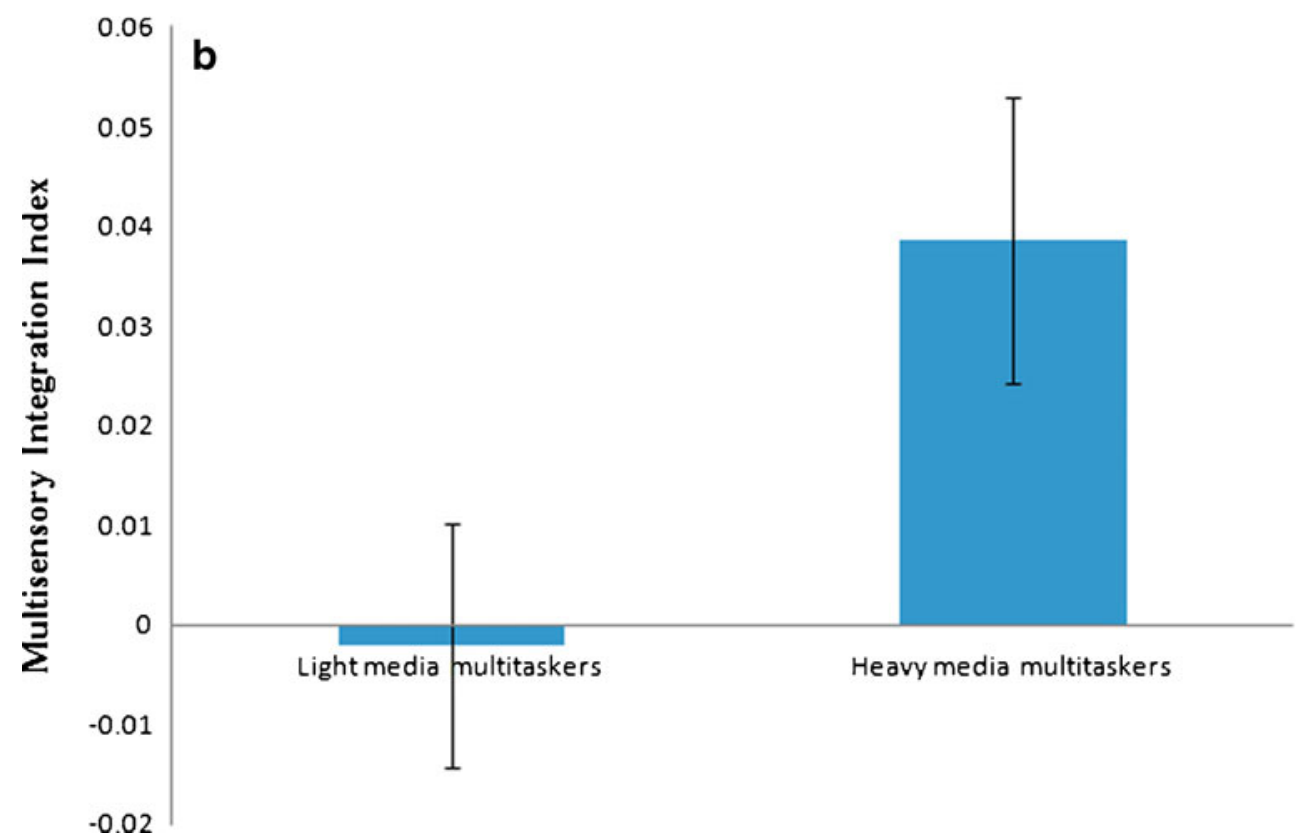

difference disappeared. It appears that heavy media multitaskers' poorer performance in the tone-absent condition was compensated for by their better multisensory integration performance (Fig. 5c). One may argue that, since the light media multitaskers performed better in the tone-absent condition, they might have suffered more from the ceiling effect and, hence, had little room to improve with the presence of a tone.

In order to test whether the ceiling effect was the primary cause of the present finding, we excluded data from participants $(N=43)$ whose accuracy was $95 \%$ or above in the tone-absent condition and conducted the correlation analyses again. If the ceiling performance for low media multitaskers was really the driving force of the effect, the positive correlation would be eliminated. Figure $5 \mathrm{~d}-\mathrm{f}$ shows the results after excluding data from the participants whose accuracy was $95 \%$ or above in the tone-absent condition. The overall pattern of results was similar, with heavy media multitaskers performing worse only in the tone-absent condition, but not in the tone-present condition, and there was a trend toward a positive correlation between the MMI and multitasking integration index. Although the trend did not reach significance, this was mainly due to the small sample size (16). Therefore, we believe that the ceiling effect was not the primary cause of the present finding.

\section{Discussion}

Research so far has revealed the detrimental aspects of media multitasking, demonstrating impairments in different 
Fig. 5 Scatterplots between participants' media multitasking index and their performance in terms of accuracy in the tone-absent condition, the tone-present condition, and the overall pip-and-pop task (multisensory integration index), before $(\mathbf{a}, \mathbf{b}, \mathbf{c})$ and after $(\mathbf{d}, \mathbf{e}, \mathbf{f}) 43$ participants whose accuracy was $95 \%$ or above in the tone-absent condition were excluded
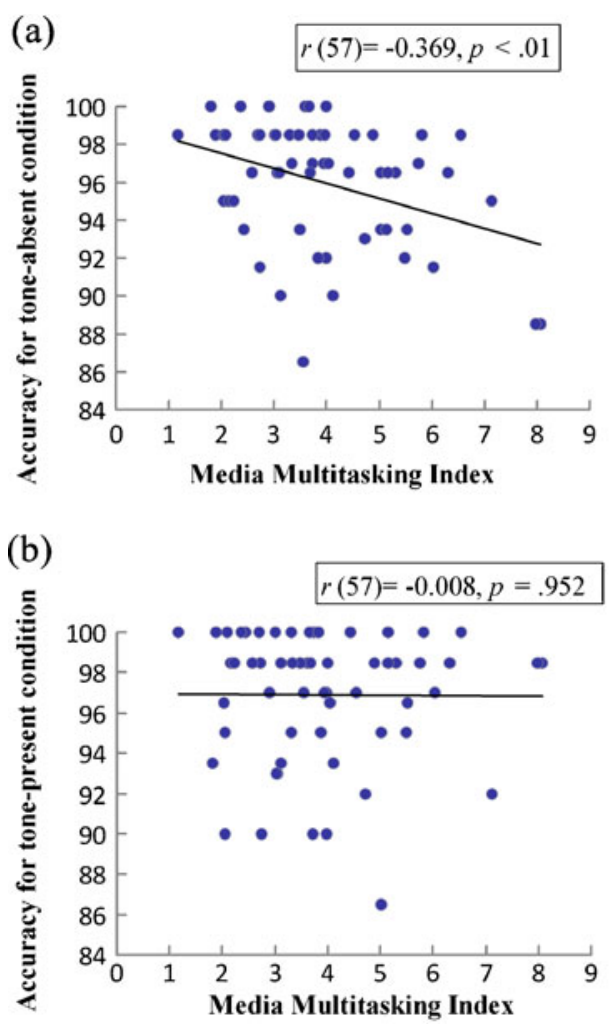

(c)

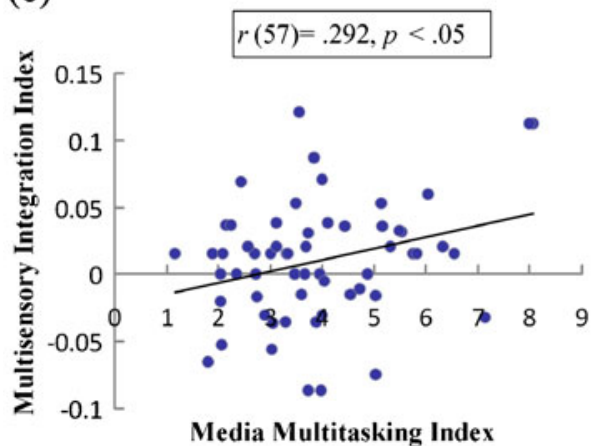

(d)

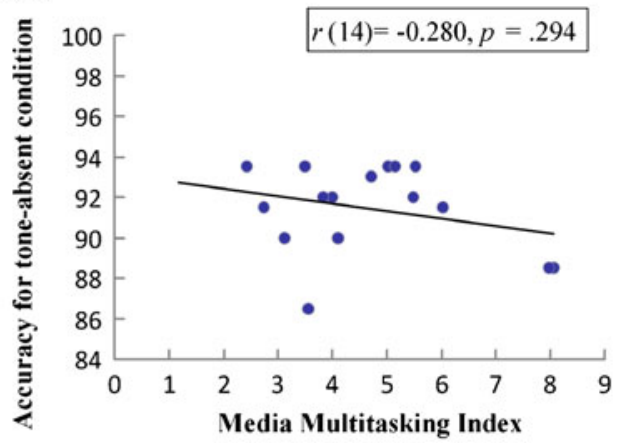

(e)

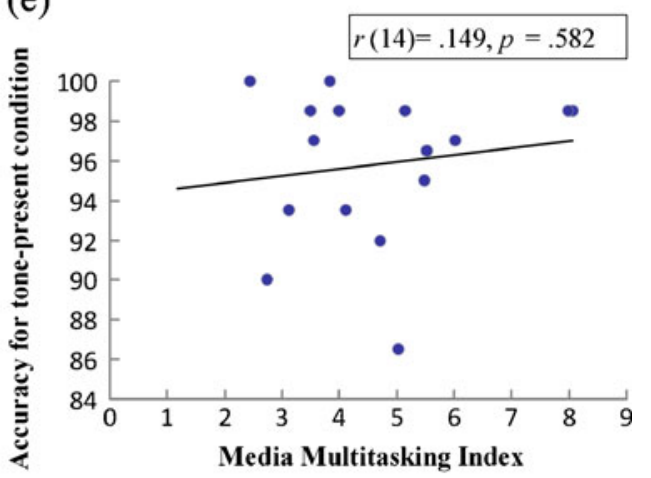

(f)

$$
r(14)=.266, p=.320
$$

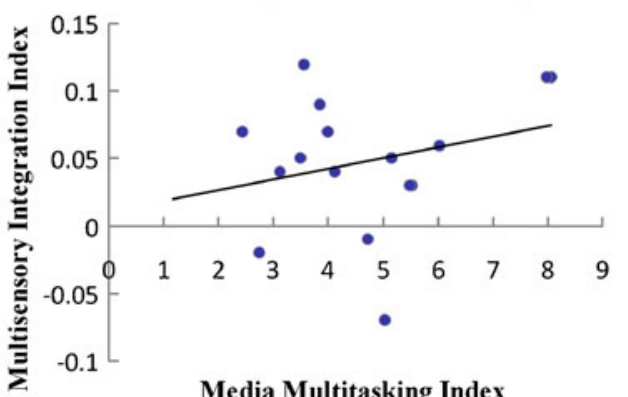

cognitive tasks in laboratories and real-world settings. This is certainly bad news for the younger generation, which embraces this multitasking lifestyle. The average MMI in the present study was 3.82 , meaning that an average undergraduate may receive information from almost four media at the same time. However, while their breadth-biased processing style may have weakened their ability to focus on information relevant to the task on hand, it may actually be beneficial in certain situations. While, in many laboratory tasks, the source of relevant information is often well defined, in reality, unexpected environmental stimuli may carry important information, which may be better captured by individuals biased toward receiving information from many different channels, rather than processing information deeply from a single channel.
The pip-and-pop paradigm used in the presenst study represents an attempt to mimic such real-world circumstances. Observers in this visual search task do not need to attend to the tones, since they are not informed of their importance in the instructions; in fact, the tones carry no information about the location, color, or orientation of the target but simply synchronize with its color changes. Yet one typically finds it easier to find the target when the tones are present, since the tones apparently are integrated with the visual target, causing the latter to pop out from the busy display. In the present study, heavy media multitaskers performed worse than light media multitaskers in the visual search task without the presence of a tone. This is consistent with the understanding that they had a bias toward breadth in their cognitive control, leading to higher difficulty in 
handling the 47 distractor line segments in the display while searching for the target. Their breadth bias, however, may have made it easier for them to utilize the unexpected auditory signal in the tone-present condition, leading to a large improvement in the presence of a tone.

One may argue that multisensory experience is not restricted to multimedia experience. Some media, such as television and the computer, offer large amounts of visual and auditory information, and one may expect improvement in multisensory integration performance as a function of experience with a single medium. It should be noted, however, that the visual and auditory information from a single medium are usually correlated (e.g., the lip movement and the speech from a character in a TV show). Information from different media (e.g., the magazine one is reading and the alert tone from a Web messenger software) usually serve different purposes and are, thus, much less often correlated. The present study focused on this aspect of multisensory integration, where one is engaged with a primary task and does not expect or even know of the utility of information coming from another channel. Future research will tell whether media multitasking experience is also associated with the ability to integrate multisensory information that is clearly relevant to the task at hand.

The use of a questionnaire to measure media multitasking is limited for a number of reasons. First, self-reports may not be an objective and accurate measure (e.g., Greenberg et al., 2005; Podsakoff \& Organ, 1986). Second, the questionnaire was concerned only with an overall quantitative measure of the extent of media multitasking, but not with more detailed measures of the different aspects involved, including the active effort involved, the overlap of task demands, the number of task switches, and so forth. A single number does not capture the potentially different types of media multitasking either. Playing background music while playing computer games, for example, may be very different from reading a textbook while searching for information on the Internet.

Although the correlation between media multitasking and multisensory integration is intriguing and we believe that media multitasking is the cause of the better multisensory integration performance, the present finding did not imply any causal relationships. Further studies may build on this finding and examine the effects of training regimens involving different types and the extent of media multitasking experience. In any case, though, the present study has highlighted an interesting possibility of the effect of media multitasking and the sensitivity of the pip-and-pop paradigm to individual differences in multisensory integration. We should consider more the possibility that multitasking may not always hurt.
Acknowledgments The authors wish to thank Dr. Erik Van der Burg for providing us with the pip-and-pop paradigm. We also thank Crystal Yuen for her assistance in data collection.

\section{References}

Armstrong, G. B., \& Chung, L. (2000). Background television and reading memory in context: Assessing TV interference and facilitative context effects on encoding versus retrieval processes. Communication Research, 27, 327-352.

Foehr, U. G. (2006). Media multitasking among American youth: Prevalence, predictors and pairings. Menlo Park, CA: Kaiser Family Foundation.

Fox, A. B., Rosen, J., \& Crawford, M. (2009). Distractions, distractions: Does instant messaging affect college students' performance on a concurrent reading comprehension task? Cyberpsychology and Behavior, 12, 51-53.

Furnham, A., \& Bradley, A. (1997). Music while you work: The differential distraction of background music on the cognitive test performance of introverts and extraverts. Applied Cognitive Psychology, 11, 445-455.

Greenberg, B. S., Eastin, M. S., Skalski, P., Cooper, L., Levy, M., \& Lachlan, K. (2005). Comparing survey and diary measures of Internet and traditional media use. Communication Reports, 18 , $1-8$.

Hembrooke, H., \& Gay, G. (2003). The laptop and the lecture: The effects of multitasking in learning environments. Journal of Computing in Higher Education, 15, 46-64.

Kirschner, P. A., \& Karpinski, A. C. (2010). Facebook and academic performance. Computers in Human Behavior, 26, $1237-1245$.

Lin, L. (2009). Breadth-biased versus focused cognitive control in media multitasking behaviors. Proceedings of the National Academy of Sciences, 106, 15521-15522.

Lin, L., Robertson, T., \& Lee, J. (2009). Reading performances between novices and experts in different media multitasking environments. Computers in the School, 26, 169-186.

Nunes, L. M., \& Recarte, M. A. (2002). Cognitive demands of handsfree-phone conversation while driving. Transportation Research, $5,133-144$.

Ophir, E., Nass, C., \& Wagner, A. D. (2009). Cognitive control in media multitaskers. Proceedings of the National Academy of Sciences, 106, 15583-15587.

Podsakoff, P. M., \& Organ, D. W. (1986). Self-reports in organizational research: Problems and prospects. Journal of Management, 12, 531-544.

Roberts, D. F., Foehr, U. G., \& Rideout, V. (2005). Generation M: Media in the lives of 8-18 year-olds. Washington, DC: Henry J. Kaiser Family Foundation.

Strayer, D. L., \& Johnston, W. A. (2001). Driven to distraction: Dual-task studies of simulated driving and conversing on a cellular telephone. Psychological Science, 12, 462-466.

Van der Burg, E., Olivers, C. N., Bronkhorst, A. W., \& Theeuwes, J. (2008). Pip and pop: Nonspatial auditory signals improve spatial visual search. Journal of Experimental Psychology. Human Perception and Performance, 34, 1053-1065.

Zhang, W., Jeong, S., \& Fishbein, M. (2010). Situational factors competing for attention: The interaction effect of multitasking and sexually explicit content on TV recognition. Journal of Media Psychology, 22, 2-13. 